\title{
Identification and characterization of Gypsophila paniculata color morphs in Sleeping Bear Dunes National Lakeshore, MI, USA
}

\author{
Marisa L Yang ${ }^{1}$, Emma Rice ${ }^{2}$, Hailee Leimbach-Maus ${ }^{2}$, Charlyn G Partridge ${ }^{\text {Corresp. } 2}$ \\ ${ }^{1}$ Environmental Science Policy and Management, University of California, Berkeley, Berkeley, California, United States \\ 2 Annis Water Resources Institute, Grand Valley State University, Muskegon, Michigan, United States \\ Corresponding Author: Charlyn G Partridge \\ Email address: partridc@gvsu.edu
}

Background. Gypsophila paniculata (baby's breath) is an invasive species found throughout much of the northwest United States and western Canada. Recently, plants exhibiting a different color morphology were identified within the coastal dunes along eastern Lake Michigan. The common baby's breath (G. paniculata) typically produces stems that are purple in color (purple morph), while the atypical morph has stems that are green-yellow (green-yellow morph). The purpose of this study was to characterize these newly identified morphs and determine if they are genetically distinct species from the common baby's breath in order to assess whether alternative management strategies should be employed to control these populations. Methods. We sequenced two chloroplast regions, rbcL and matK, and one nuclear region, ITS2, from the purple morphs and green-yellow morphs collected from Sleeping Bear Dunes National Lakeshore, MI, USA (SBDNL). Sequences were aligned to reference sequences from other Gypsophila species obtained from the Barcode of Life (BOLD) and GenBank databases. We also collected seeds from wild purple morph and wild green-yellow morph plants in SBDNL. We grew the seeds in a common garden setting and characterized the proportion of green-yellow individuals produced from the two color morphs after five-months of growth. Results. Phylogenetic analyses based upon rbcL, matK, and ITS2 regions suggest that the two color morphs are not distinct species and they both belong to $G$. paniculata. Seeds collected from wild green-yellow morphs produced a significantly higher proportion of green-yellow individuals compared to the number produced by seeds collected from wild purple morphs. However, seeds collected from both color morphs produced more purple morphs than green-yellow morphs. Discussion. Based upon these results, we propose that the two color morphs are variants of $G$. paniculata. Given the significant difference in the number of green-yellow morphs produced from the seeds of each morph type, we also suggest that this color difference has some genetic basis. We propose that current management 
continue to treat the two color morphs in a similar manner in terms of removal to prevent the further spread of this species. 
1 Identification and characterization of Gypsophila paniculata color morphs in Sleeping Bear

2 Dunes National Lakeshore, MI, USA.

3

4 Marisa L. Yang ${ }^{1}$, Emma Rice ${ }^{2}$, Hailee Leimbach-Maus ${ }^{2}$, and Charlyn G. Partridge ${ }^{2}$

$6 \quad{ }^{1}$ Environmental Science Policy, and Management, University of California, Berkeley, 260

7 Mulford Hall, Berkeley, CA 94720, email: marisayang79@gmail.com

$8 \quad{ }^{2}$ Annis Water Resources Institute, 740 W. Shoreline Dr., Muskegon, MI 49441, email:

9 riceemm@mail.gvsu.edu; hailee.pavisich@gmail.com

11 Corresponding author: Charlyn Partridge, Annis Water Resources Institute, Grand Valley State

12 University, 740 W. Shoreline Dr., Muskegon, MI; 49441, email: partride@gvsu.edu

\section{Abstract}

\section{Background.}

16 Gypsophila paniculata (baby's breath) is an invasive species found throughout much of the

17 northwest United States and western Canada. Recently, plants exhibiting a different color

18 morphology were identified within the coastal dunes along eastern Lake Michigan. The common

19 baby's breath (G. paniculata) typically produces stems that are purple in color (purple morph),

20 while the atypical morph has stems that are green-yellow (green-yellow morph). The purpose of

21 this study was to characterize these newly identified morphs and determine if they are genetically

22 distinct species from the common baby's breath in order to assess whether alternative

23 management strategies should be employed to control these populations. 
25 Methods. We sequenced two chloroplast regions, rbcL and matK, and one nuclear region, ITS2,

26 from the purple morphs and green-yellow morphs collected from Sleeping Bear Dunes National

27 Lakeshore, MI, USA (SBDNL). Sequences were aligned to reference sequences from other

28 Gypsophila species obtained from the Barcode of Life (BOLD) and GenBank databases. We also

29 collected seeds from wild purple morph and wild green-yellow morph plants in SBDNL. We

30 grew the seeds in a common garden setting and characterized the proportion of green-yellow

31 individuals produced from the two color morphs after five-months of growth.

32

33 Results. Phylogenetic analyses based upon $\mathrm{rbcL}$, matK, and ITS2 regions suggest that the two

34 color morphs are not distinct species and they both belong to G. paniculata. Seeds collected from

35 wild green-yellow morphs produced a significantly higher proportion of green-yellow

36 individuals compared to the number produced by seeds collected from wild purple morphs.

37 However, seeds collected from both color morphs produced more purple morphs than green-

38 yellow morphs.

Discussion. Based upon these results, we propose that the two color morphs are variants of $G$. paniculata. Given the significant difference in the number of green-yellow morphs produced

42 from the seeds of each morph type, we also suggest that this color difference has some genetic

43 basis. We propose that current management continue to treat the two color morphs in a similar

44 manner in terms of removal to prevent the further spread of this species. 


\section{Introduction}

The Great Lakes sand dunes comprise the most extensive freshwater dune complex in the world, stretching over $1,000 \mathrm{~km}^{2}$ in Michigan alone. Within northwest Michigan, the sand dunes ecosystem is vital both environmentally and economically. It is home to a number of threatened and endangered species, including piping plover (Charadrius melodus) and Pitcher's thistle

52 53

(Cirsium pitcheri). Colonization of invasive species in this region has the potential to significantly alter the biological composition of these native communities (Leege and Murphy, 2001; Emery et al. 2013). One invasive species of significant concern is the perennial baby's breath (Gypsophila paniculata). In 2015, baby's breath was listed by the Michigan Department of Natural Resources (DNR) as a "priority" invasive species for detection and control in Michigan's northern lower peninsula (DNR, 2015). Since its colonization in the region it has spread along a $260 \mathrm{~km}$ stretch of the Michigan shoreline. Baby's breath produces a large taproot system that can extend down to 4 meters in depth, which likely helps it outcompete native vegetation for limited resources (Darwent \& Coupland, 1966; Karamanski, 2000). In addition, while many of the vulnerable and endangered plant species in these areas are seed limited, (e.g., Pitcher's thistle produces approximately 50-300 seeds per plant total or 'per lifetime' (Bevill et al., 1999)), baby's breath can produce up to 14,000 seeds per plant annually (Stevens, 1957), effectively outcompeting native species in terms of overall yield. This has led to baby's breath composing approximately $50-80 \%$ of the ground cover in some areas (Karamanski, 2000; Emery et al., 2013).

One concern with current management efforts is that anecdotal evidence suggests there may be a new baby's breath variant within the Michigan dune system. In 2011 and 2012 The Nature Conservancy (TNC) removal crews reported baby's breath plants with different character 
70 traits than what is commonly observed (TNC, 2014). The atypical morph has stems and leaves

71 that are lighter in color and more yellow than the common G. paniculata purple morph (Figure

72 1a-c). The purple morph has a thick taproot (4-7 $\mathrm{cm}$ in diameter) just below the caudex that

73 remains unbranched for approximately $60-100 \mathrm{~cm}$ (Darwent \& Coupland, 1966). Severing just

74 below the intersection of the caudex and the taproot is where manual removal efforts target to

75 limit regrowth. However, TNC removal crews suggested that the atypical green-yellow morph's

76 root system seemed to be more diffuse, making it harder to identify a primary taproot and thus,

77 harder to sever without the potential for regrowth (TNC, 2014). Currently, these green-yellow

78 morphs are treated with herbicide application (glyphosate) when observed; however, if this is a

newly invaded baby's breath species and it continues to spread into areas where threatened or

80 endangered species are present, removal methods will be a primary concern and alternative

81 management strategies may need to be considered for these populations.

82 One of the first steps toward adapting current management strategies for this invasive is

83 to identify whether the green-yellow morph is a genetically distinct species from the purple

84 morph. While G. paniculata is the dominant invasive baby's breath species in northwest

85 Michigan, a number of other species have been introduced to North America and, specifically,

86 the Great Lakes region (Pringle, 1976, Voss \& Reznicek, 2012). For example, G. elegans, G.

87 scorzonerifolia, G. muralis, and G. acutifolia have been collected within Michigan (Pringle,

88 1976; Reznicek et al., 2011; Voss \& Reznicek, 2012), and G. perfoliata is reported to have

89 become naturalized in the United States (Pringle, 1976). G. muralis is an annual and has a very

90 distinct morphology compared to the other Gypsophila species identified around the Great

91 Lakes. It typically only reaches $5-20 \mathrm{~cm}$ in height, has linear leaves, and commonly produces

92 white to pink flowers (Barkoudah, 1962). G. elegans, also an annual, is commonly sold in this 
93 region in commercial wildflower packets. It typically has a smaller taproot compared to $G$.

94 paniculata, and its coloration can be similar to that observed for the green-yellow morph. $G$.

95 scorzonerifolia and G. acutifolia are perennials and specimens of these species have been

96 collected in counties within the Great Lakes dune system that also contain G. paniculata

97 infestations (Voss, 1957; Pringle, 1976). Both G. scorzonerifolia and G. acutifolia have a deep

98 taproot and are similar in height to G. paniculata, and thus, can superficially resemble $G$.

99 paniculata (Voss, 1957; Pringle, 1976). However, both can be distinguished from G. paniculata

100 in that their leaves tend to be longer and wider, and the pedicels and calyces are glandular as

101 opposed to glabrous in G. paniculata (Voss, 1957; Pringle, 1976). Given the potential for these

102 other species to invade the fragile habitat of the Michigan dune system, the goal of this work was

103 to characterize the genetic relationship between the newly recognized green-yellow morph and

104 the common purple morph to determine if they are the same species.

106 Methods and materials

107 DNA Extraction, Amplification, and Sequencing

108 We collected leaf tissue from 1 green-yellow morph and 16 purple morphs in 2016 and

109 an additional 15 green-yellow morphs in 2017 from Sleeping Bear Dunes National Lakeshore

110 (SBDNL), Empire, MI, USA (specifically: $44.884941 \mathrm{~N}, 86.062111 \mathrm{~W}$ and $44.875302 \mathrm{~N}$,

$11186.056821 \mathrm{~W}$ ). Plant tissue collections were approved by the National Parks Service (permit ID

112 SLBE-2015-SCI-0013). Leaf tissue was dried in silica gel until DNA extractions could take

113 place. DNA was extracted using a Qiagen DNeasy Plant Mini Kit (Qiagen, Hilden, Germany).

114 After extraction, the DNA samples were placed through Zymo OneStep PCR inhibitor removal

115 columns (Zymo, Irvine, CA) to remove any secondary metabolites that might inhibit PCR 
116 amplification. The DNA for each sample was then quantified using a NanoDrop 2000

117 (ThermoFisher, Waltham, MA).

118 The DNA of green-yellow morphs and purple morphs was amplified at three genetic

119 regions: large subunit of the ribulose-bisphosphate carboxylase gene ( $\mathrm{rbcL}$ ), maturase $\mathrm{K}$ (matK), 120 and internal transcribed spacer 2 (ITS2). A combination of ITS region and matK have been used

121 to differentiate between other Gypsophila species (specifically, G. elegans and G. repens) in

122 previous studies (Fior et al., 2006). The rbcL region was amplified using rbcL 1F and rbcL 724R

123 primers (Fay et al., 1997), matK was amplified using matK 390F and matK 1440R primers (Fior

124 et al., 2006), and the ITS2 region was amplified using ITS2 2SF and ITS2 S3R primers (Chen et

125 al., 2010). PCR reactions for all loci consisted of $1 \mathrm{X}$ Taq Buffer, $2.0 \mathrm{mM} \mathrm{MgCl} 2,0.3 \mu \mathrm{M}$ dNTP,

$1260.08 \mathrm{mg} / \mathrm{mL}$ BSA, $0.4 \mu \mathrm{M}$ forward primer, $0.4 \mu \mathrm{M}$ reverse primer, and 0.5 units of Taq

127 polymerase in a $20 \mu \mathrm{L}$ reaction volume. The thermal cycle protocols consisted of the following:

128 for rbcL, an initial denaturing step of $95^{\circ} \mathrm{C}$ for 2 minutes, followed by 35 cycles of $94^{\circ} \mathrm{C}$ for 1

129 minute, $55^{\circ} \mathrm{C}$ for 30 seconds, and $72^{\circ} \mathrm{C}$ for 1 minute. A final elongation step was performed at

$13072^{\circ} \mathrm{C}$ for 7 minutes. For matK, the thermal profile consisted of 26 cycles of $94^{\circ} \mathrm{C}$ for 1 minute,

$13148^{\circ} \mathrm{C}$ for 30 seconds, and $72^{\circ} \mathrm{C}$ for 1 minute, followed by a final elongation step at $72^{\circ} \mathrm{C}$ for 7

132 minutes. For ITS2, an initial denaturing step of $95^{\circ} \mathrm{C}$ for 2 minutes was applied, followed by 35

133 cycles of $95^{\circ} \mathrm{C}$ for 30 seconds, $50^{\circ} \mathrm{C}$ for 30 seconds, $72^{\circ} \mathrm{C}$ for 1.5 minutes, and a final elongation

134 step of $72^{\circ} \mathrm{C}$ for 8 minutes. Successful amplification was checked by running the PCR product on

135 a 2\% agarose gel stained with ethidium bromide. PCR reactions were then cleaned using

136 ExoSAP-IT PCR Product Cleanup Reagent (ThermoFisher, Waltham, MA). Sequencing

137 reactions were performed with the forward and reverse primers for each of the three regions.

138 Sequencing reactions were cleaned using a Sephadex column (GE Healthcare Life Science, 
139 Marlborough, MA) and sequenced on an ABI Genetic BioAnalyzer 3130xl (Applied Biosystems,

140 Foster City, CA). Out of the 16 green-yellow morphs a total of 13 were successfully sequenced

141 for rbcL, 13 were successfully sequenced for matK, and 14 were successfully sequenced for

142 ITS2. For the purple morphs a total of 15, 12, and 15 individuals were successfully sequenced

143 for rbcL, matK and ITS2, respectively.

144 Reference sequences for rbcL, matK, and ITS2 of other Gypsophila spp. were

145 downloaded from either the Barcode of Life Database (BOLD) (http://www.barcodeoflife.org) or

146 GenBank (https://www.ncbi.nlm.nih.gov/genbank/). We primarily focused on Gypsophila

147 species with reported occurrences within the United States, but also incorporated other species if

148 their information was available on BOLD. Sequences of the three regions were not always

149 available for the same species, thus for rbcL these included G. paniculata, G. elegans, G.

150 fastigiata, G. scorzonerifolia, G. perfoliata, and G. muralis. For matK the species included G.

151 paniculata, G. elegans, G. fastigiata, G. scorzonerifolia, G. perfoliata, G. muralis, G. altissima,

152 and G. repens. For the IST2 region, the species included G. paniculata, G. elegans, G.

153 scorzonerifolia, G. perfoliata, G. repens, and G. acutifolia. Because sequences for all three

154 regions were not available for all species, our merged phylogeny only contained G. paniculata,

155 G. elegans, G. scorzonerifolia, and G. perfoliata reference sequences. The accession numbers

156 and sequences for all reference species are provided in Supplemental Table 1. All FASTA files

157 corresponding to these data will be deposited in the Dryad database and sequences have been

158 submitted to GenBank (ITS2: MG385003-385031, matK: MG603322-603346, rbcL:

159 MG547346-547373).

160

161 
162

163

164

165

166

167

168

169

170

171

172

173

174

175

176

177

178

179

180

181

182

183

184

\section{Alignment and Phylogenetic Analysis}

All successful sequences from our field samples, as well as sequences for other

Gyposphila species reference sequences obtained from BOLD or GenBank, were imported into the program MEGA7 (version 7.0.14) (Kumar et al., 2016) and sequences for each of the three regions were aligned both individually and with all sequences combined using Muscle (Edgar, 2004). The total number of base pairs aligned and analyzed for each region included: 427 base pair (bp) for rbcL, 702 bp for matK, $201 \mathrm{bp}$ for ITS2, and $1330 \mathrm{bp}$ for the three regions combined. All alignment parameters were kept at their default settings. Once aligned, we used MEGA7 to identify the most appropriate substitution model (rbcL: Jukes-Cantor, matK: Tamura 3-parameter, ITS2: Jukes-Cantor, all genes combined: Tamura 3-parameter with gamma distribution). We then created phylogenetic trees using a maximum-likelihood (ML) approach with 500-replicated bootstrap analyses, as well as using neighbor joining, and parsimony models.

We also constructed a TCS haplotype network (Clement et al., 2002) based upon the combined sequences using the statistical parsimony approach (Templeton et al., 1992) in the program PopART (v 1.7, http://popart.tago.ac.nz).

\section{Color Morph Germination}

On May 8, 2018 we planted a total of 207 seeds collected from mature purple morphs and 255 seeds collected from mature green-yellow morphs from SBDNL. For the purple morph seeds, these were collected from a total of 14 plants that were sampled in 2016 (average 15 seeds per plant) and 7 plants that were sampled in 2017 (average 1.7 seeds per plant). For the greenyellow morphs, these seeds were collected from a total of 17 plants (15 seeds per plant) in 2017. Plants were grown in the GVSU - Allendale greenhouse from May until August, 2018. The 
185 greenhouse was on a 17 hour light/ 7 hour dark cycle. The average day temperature was $21^{\circ} \mathrm{C}$

186 and night temperature was $15^{\circ} \mathrm{C}$. In August the plants were transported to the greenhouse at

187 AWRI-GVSU where they were allowed to grow until October 15, 2018. The greenhouse at

188 AWRI-GVSU has no external lighting source or temperature controls, and thus more closely

189 resembled seasonal day/night and temperature cycles. Plants were sampled after a decrease in

190 temperature occurred (from a high of $23{ }^{\circ} \mathrm{C}$ on October 10,2018 to a high of $11{ }^{\circ} \mathrm{C}$ on October

191 15, 2018). Previous greenhouse observations have found that the differences between the purple

192 and green-yellow morphs can be best detected after a sudden drop in temperature (personal

193 observation, CGP). On October 15, we characterized the color of all individuals that successfully

194 germinated and survived over the 5-month period. We used a chi-square analysis in the R

195 statistical package (v3.5.1) to determine if germination success differed between seeds from the

196 two color morphs and whether the proportion of seeds that developed into green-yellow morphs

197 significantly differed between seeds collected from mature purple and mature green-yellow 198 plants.

\section{Results}

200 Our results indicate that the green-yellow morph identified in SBDNL is not a genetically

201 distinct species from the common purple found throughout SBDNL. The rbcL, matK, ITS2, and

202 combined dataset showed similar patterns with both the green-yellow morphs and the purple

203 morphs clustering together. The phylogenies constructed from rbcL and matK independently

204 show that the two color morphs cluster separately from G. fastigata, G. elegan, G. muralis, and

205 G. repens. For the rbcL locus, the relationship of the color morphs to G. paniculata and G.

206 scorzonerifolia was not resolved. Additionally, when we only examined the matK gene, the color

207 morphs clustered separately but within a clade that also included G. altissima, G. scorzonerifolia, 
208 and G. paniculata. The ITS2 region was able to provide more resolution between G. paniculata,

209 G. scorzonerifolia, G. acutifolia, and the color morphs, with the color morphs clustering with $G$.

210 paniculata (Figures 2-4; Figures S1-8) and separately from the G. scorzonerifolia and G.

211 acutifolia clade. The same pattern was observed when all regions were analyzed together (Figure

212 5), with the exception that this phylogeny did not include G. acutifolia. In addition, the TCS

213 haplotype network shows that the purple and green-yellow morphs have shared haplotypes.

214 These two haplotypes are only one mutation away from one another and the G. paniculata

215 reference, while the next closest species, G. scorzonerifolia is 15 mutations away (Figure 6).

216 This further suggests that both color morphs are G. paniculata.

217 Of the regions analyzed for the green-yellow morphs, purple morphs, and reference

218 sequences, rbcL was the most conserved sequence with an overall mean genetic distance $(d)=$

2190.004 , followed by matK $(d=0.015)$ and ITS2 $(d=0.038)$. For the ITS2 region, there were six

220 purple morphs and one green-yellow morph that clustered together inside the G. paniculata

221 branch (Figure $4 \& 5$ ). Further examination of the electropherograms for these individuals show

222 that they are likely heterozygous at position 138 of our aligned sequence and amplification bias

223 of the 'A' single nucleotide polymorphism (SNP) over the allele containing the ' $\mathrm{G}$ ' SNP is

224 driving this pattern.

225

226

Color Morph Germination

Out of the 207 seeds that were collected from mature purple morphs and planted in the

228 greenhouse, 82 successfully germinated and survived over the 5 month period (39.6\%). Out of

229 these 82 plants, only one green-yellow morph was produced (1.2\%), while the remaining seeds

230 all produced purple morphs. Out of the 255 seeds collected from mature green-yellow morphs 
231 and planted in the greenhouse, 105 successfully germinated and survived over the 5 month

232 period $(41.2 \%)$. This was not significantly different than the proportion of plants that

233 successfully germinated from the purple morph seeds $\left(\chi^{2}=0.06, \mathrm{df}=1, \mathrm{p}=0.81\right)$. Of the 105

234 successfully germinated seeds from the green-yellow morph plants, 12 developed into green-

235 yellow morph plants (11.4\%), 91 developed into purple morphs plants $(86.7 \%)$, and two plants

236 could not be determined (they appeared to be green-yellow morphs but displayed some dark

237 spots on the stem). The proportion of seeds that produced green-yellow individuals significantly

238 differed between seeds collected from mature green-yellow morphs and seeds collected from

239 mature purple morphs $\left(\chi^{2}=5.9, \mathrm{df}=1, \mathrm{p}=0.015\right)$.

241 Discussion

242 Overall, our data suggest that the green-yellow morph is not a genetically distinct species

243 from the purple morph, and that both morphs are G. paniculata. For all molecular markers used,

244 the green-yellow and the purple color morphs grouped together. RbcL, matK, and ITS2 are

245 common 'barcode' regions used to delineate plant species (Newmaster et al., 2006; Group et al.,

246 2009; Chen et al., 2010; Yao et al., 2010; Stoeckle et al., 2011) and when used in combination

247 they provided adequate resolution to separate out the Gypsophila species included in this study.

248 In our data set, rbcL and matK worked well to separate our color morphs from G. elegans, G.

249 muralis and both of these species have been reported to occur in the Great Lakes region

250 (Reznicek et al., 2011; Voss \& Reznicek 2012). While the morphology of G. muralis is very

251 distinct from the color morphs in SBDNL, it was initially thought that G. elegans shared some

252 similar traits to that originally described by the TNC removal crews and was a potential 
253 candidate species for the green-yellow color morph. Based upon these results, this is clearly not 254 the case.

255 The phylogeny based on ITS2 region and the combined sequences provided the best 256 resolution for assigning the relationship of our Gypsophila species. Like the rbcL and matK 257 phylogenies, all the purple and green yellow morphs grouped together. For this region, the color 258 morphs also grouped within the same clade as the G. paniculata reference sequence. While $G$. 259 scorzonerifolia and G. acutifolia have also been recorded in the Great Lakes regions (Pringle, 1976), and have a similar general phenotype as G. paniculata, these species were clearly within a

261 distinct clade that was separate from the two color morphs. Similarly, while G. perfoliata has

262 been reported to be naturalized in North America (Pringle, 1976), it grouped outside of the $G$.

263 paniculata and color morph cluster.

Our greenhouse germination study showed that seeds collected from mature green-yellow

265 morphs produced a significantly higher proportion of green-yellow individuals than seeds 266 collected from mature purple morphs. Of the seeds collected from mature green-yellow morphs

$26711 \%$ resulted in green-yellow morphs, while only $1 \%$ of seeds from mature purple morphs 268 resulted in green-yellow morphs. However, seeds from both color morphs primarily produced 269 purple morphs. The mechanism driving the color difference between the purple and green270 morphs is currently unknown. Within SBDNL, the purple morph is the most common form, with 271 green-yellow individuals found interspersed in a couple of locations throughout the dunes

272 (personal observation, CGP/HLM/ER). The largest observed group of green-yellow morphs

273 consists of a few hundred plants clumped within approximately an acre-sized area and

274 interspersed throughout large groups of purple morphs. Based upon the dispersal patterns of the 275 two morphs throughout the dunes, and our germination results, the color difference observed 
276 does not appear to be solely environmentally driven, and likely has a genetic component.

277 Potential candidate genes that could be influencing these color differences include those involved

278 in the anthocyanin pathway, which influences red - purple coloration in a number of plants

279 (Asen et al., 1972; de Pascual-Teresa et al., 2002; Abdel-Aal et al., 2006). In addition,

280 anthocyanin can rapidly accumulate in the shoots of plants following cold exposure (Leng et al.,

281 2000), and we have observed an increase in the amount of purple coloration in the purple morphs

282 as temperatures decrease (personal observation, CGP). Further work will begin to elucidate the

283 specific mechanism influencing this color difference in invasive G. paniculata populations, as

284 well as to explore whether this color variation drives functional differences between the morphs.

285 Taken together, these data show that the purple and green-yellow morph within SBDNL

286 are the same species, and that species is G. paniculata. One concern with the green-yellow

287 morph initially noted by TNC removal crews was that the taproot tended to be more diffuse than

288 the purple morph, potentially making manual removal of these plants less effective. However, we

289 have not noted differences in the taproot structure between these two morphs when grown under

290 controlled conditions (Figure 7). Additionally, our lab's personal observations (CGP, HLM) in

291 the field have not found any indication that large differences in root structure occur between

292 mature plants of the two color morphs. Therefore, current management approaches for these

293 populations should be maintained to control the further spread of G. paniculata throughout the

294 Michigan coastal dune system.

295

296 Conclusions

297 Our data show that both the purple and green-yellow color morph of baby's breath in

298 Sleeping Bear Dunes National Lakeshore are G. paniculata and the observed color differences 
299 likely have some genetic basis. Based on this current information, we recommend that these

300 color morphs continue to be managed in a similar manner and that distinct management

301 strategies do not need to be established at this time.

302

303 Acknowledgements

304 We would like to thank Shaun Howard from The Nature Conservancy for his help in identifying 305 the green-yellow morph and Benjamin Giffin for his help with sequencing analysis. We would 306 also like to thank Kurt Thompson and Doug Haywick for assisting with the figure construction,

307 and Alexis Hoskins and Doug Haywick for helping with planting.

308

309 References

310 Abdel-Aal ESM, Young JC, Rabalski I. 2006. Anthocyanin composition in black, blue, pink, 311 purple, and red cereal grains. Journal of Agriculture Food Chemistry 54(13): 4696-4704.

312 Asen S, Stewart RN, Norris KH. 1972. Co-pigmentation of anthocyanins in plant tissues and its 313 effect on color. Phytochemistry 11(3): 1139-1144

314 Barkoudah YI. 1962. Revision of Gypsophila, Bolanthus, Ankyropetalum and Phryna. Wentia 9: $315 \quad 1-203$.

316 Bevill RL, Louda SM, Stanforth LM. 1999. Protection from natural enemies in managing rare 317 plant species. Conservation Biology 13(6): 1323-1331.

318 Chen S, Yao H, Han J, Liu C, Song J, Shi L, Zhu Y, Ma X, Gao T, Pang X, Luo K, Li X, Jai X,

319 Lin Y, Leon C. 2010. Validation of the ITS2 region as a novel DNA barcode for

320 identifying medicinal plant species. PloS One 5(1): e8613. doi:

321 10.1371/journal.pone.0008613L 
322 Clement M, Snell Q, Walker P, Pondosa D, Crandall KA. 2002. TCS: Estimating gene

323

324

325

326

327

328

329

330

331

332

333

334

335

336

337

338

339

340

341

342

343

genealogies. Parallel and Distributed Processing Symposium, International Proceedings, 2,184 .

de Pascual-Teresa S, Santos-Buelga C, Rivas-Gonzalo JC. 2002. LC-MS analysis of anthocyanins from purple corn cob. Journal of the Science of Food and Agriculture 82(9): 1003-1006.

Darwent AL, Coupland RT. (1966). Life history of Gypsophila paniculata. Weeds 14(4): 313318.

Department of Natural Resources (DNR). 2015. Michigan Invasive Species Grant Program Handbook. http://www.michigan.gov/documents/dnr/2015-MISGPhndbk_491809_7.pdf?source=govdelivery

Emery SM, Doran PJ, Legge JT, Kleitch M, Howard S. 2013. Aboveground and belowground impacts following removal of the invasive species baby's breath (Gypsophila paniculata) on Lake Michigan sand dunes. Restoration Ecology 21(4): 506-514.

Fay MF, Swensen SM, Chase MW. 1997. Taxonomic affinities of Medusagyne oppositifolia (Medusagynaceae). Kew Bulletin 52(1): 111-120.

Fior S, Karis PO, Casazza G, Minuto L, Sala F. 2006. Molecular phylogeny of the Caryophyllaceae (Caryophyllales) inferred from chloroplast matK and nuclear rDNA ITS sequences. American Journal of Botany 93(3): 399-411.

Group CPW, Hollingsworth PM, Forrest LL, Spouge JL, Hajibabaei M, Ratnasingham S, van der Bank M, Chase MW, Cowan RS, Erickson DL, Fazekas AJ. 2009. A DNA barcode for land plants. Proceedings of the National Academy of Sciences 106(31): 12794-12797. 
344 Jukes TH, Cantor CR. 1969. Evolution of protein molecules. In: Munrio HN, ed. Mammalian Protein Metabolism. New York: Academic Press. pp. 21-132

346 Karamanski TJ. 2000. Nationalized Lakeshore: The Creation and Administration of Sleeping Bear Dunes National Lakeshore. Online Book. National Park Service, Department of the Interior. http://www.cr.nps.gov/history/online_books/slbe/

Kumar S, Stecher G, Tamura K. 2016. MEGA7: Molecular Evolutionary Genetics Analysis version 7.0 for bigger datasets. Molecular Biology and Evolution 33(7): 1870-1874.

Leege LM, Murphy PG. 2001. Ecological effects of the non-native Pinus nigra on sand dune communities. Canadian Journal of Botany 79(4): 429-437.

Leng P, Itamura H, Yamamura H, \& Deng XM. 2000. Anthocyanin accumulation in apple and peach shoots during cold acclimation. Scientia Horticulturae, 83(1): 43-50.

Newmaster SG, Fazekas AJ, Ragupathy S. 2006. DNA barcoding in land plants: evaluation of rbcL in a multigene tiered approach. Botany 84(3): 335-341.

Pringle JS. 1976. Gypsophila scorzonerifolia (Caryophyllaceae), a naturalized species in the Great Lakes region. Michigan Botanist 15: 215-219.

Reznicek AA, Voss EG, Walters BS. February. 2011. Michigan Flora Online. University of Michigan. Web. March 6, 2019. https://michiganflora.net/genus.aspx?id=Gypsophila.

Stevens OA. 1957. Weights of seeds and numbers per plant. Weeds 5: 46-55.

Stoeckle MY, Gamble CC, Kirpekar R, Young G, Ahmed S, Little DP. 2011. Commercial teas highlight plant DNA barcode identification successes and obstacles. Scientific reports 1: 42. 
365 Tamura K. 1992. Estimation of the number of nucleotide substitutions when there are strong 366 transition-transversion and G $+\mathrm{C}$-content biases. Molecular Biology and Evolution 9(4):

367 $678-687$

368 Templeton AR, Crandall KA, Sing CF. 1992. A cladistics analysis of phenotypic association 369 with haplotypes inferred from restriction endonuclease mapping and DNA sequence data. III. Cladogram estimation. Genetics 132: 619-633.

371 The Nature Conservancy (TNC). 2014. Lake Michigan Coastal Dunes Restoration Project. 2013

372 Field Season Report. The Nature Conservancy and Partners, Lansing, Michigan

373 Voss, EG. 1957. Observations on the Michigan flora-VI. Distribution records of some 374 angiosperms new, rare, or misinterpreted in the state. Brittonia 9(2): 83-101.

375 Voss EG, Reznicek, AA. 2012. Field manual of Michigan flora. Ann Arbor: University of $376 \quad$ Michigan Press.

377 378

379

380

381

382
Yao H, Song J, Liu C, Luo K, Han J, Li Y, Pang X, Xu H, Zhu Y, Xiao H, \& Chen S. 2010. Use of ITS2 region as the universal DNA barcode for plants and animals. PloS One 5(10): e13102. 


\section{Figure Legends}

384 Figure 1: (A) Green-yellow morph, (B) common purple morph and (C) stem of the green-yellow 385 and purple baby's breath morph found in Sleeping Bear Dunes National Lakeshore.

387 Figure 2: Phylogenetic analysis of the purple and green-yellow baby's breath color morphs in 388 relationship to other Gypsophila species based on the rbcL region. The evolutionary history was 389 inferred using maximum likelihood methods. For this analysis, we used a Jukes Cantor (JC) 390 model of molecular evolution (Jukes and Cantor, 1969).

391

392 Figure 3: Phylogenetic analysis of the purple and green-yellow baby's breath color morphs in

393 relationship to other Gypsophila species based on the matK region. The evolutionary history was

394 inferred using maximum likelihood methods. For the analysis, we used a Tamura 3-parameter 395 (T92) model of molecular evolution with uniform distribution (Tamura, 1992).

397 Figure 4: Phylogenetic analysis of the purple and green-yellow baby's breath color morphs in 398 relationship to other Gypsophila species based on the ITS2 region. The evolutionary history was 399 inferred using maximum likelihood methods. For the analysis we used a Jukes Cantor (JC) 400 model of molecular evolution (Jukes and Cantor, 1969).

401

402 Figure 5: Phylogenetic analysis of the purple and green-yellow baby's breath color morphs in 403 relationship to other Gypsophila species based on the rbcL, matK, and ITS2 regions combined.

404 The evolutionary history was inferred using maximum likelihood methods. For the analysis, we 
405 used a Tamura 3-parameter (T92) model of molecular evolution with gamma distribution 406 (Tamura, 1992).

407

408 Figure 6: A TCS haplotype network based on rbcL, matK and ITS2 combined for the purple and 409 green-yellow baby's breath color morphs and the G. paniculata, G. elegans, G. perfoliata, and 410 G. scorzonerifolia reference sequences. The size of the ovals correspond to the haplotype

411 frequency. The hash marks represent the number of mutations between each haplotype.

412

413 Figure 7: (A) Green-yellow morph, and (B) common purple morph after 5-months in the GVSU 414 greenhouse. Note the similarity in taproot structure between the two plants.

415

416

417 
Figure 1

(A) Green-yellow morph, (B) common purple morph and (C) stem of the green-yellow and purple baby's breath morph found in Sleeping Bear Dunes National Lakeshore. 

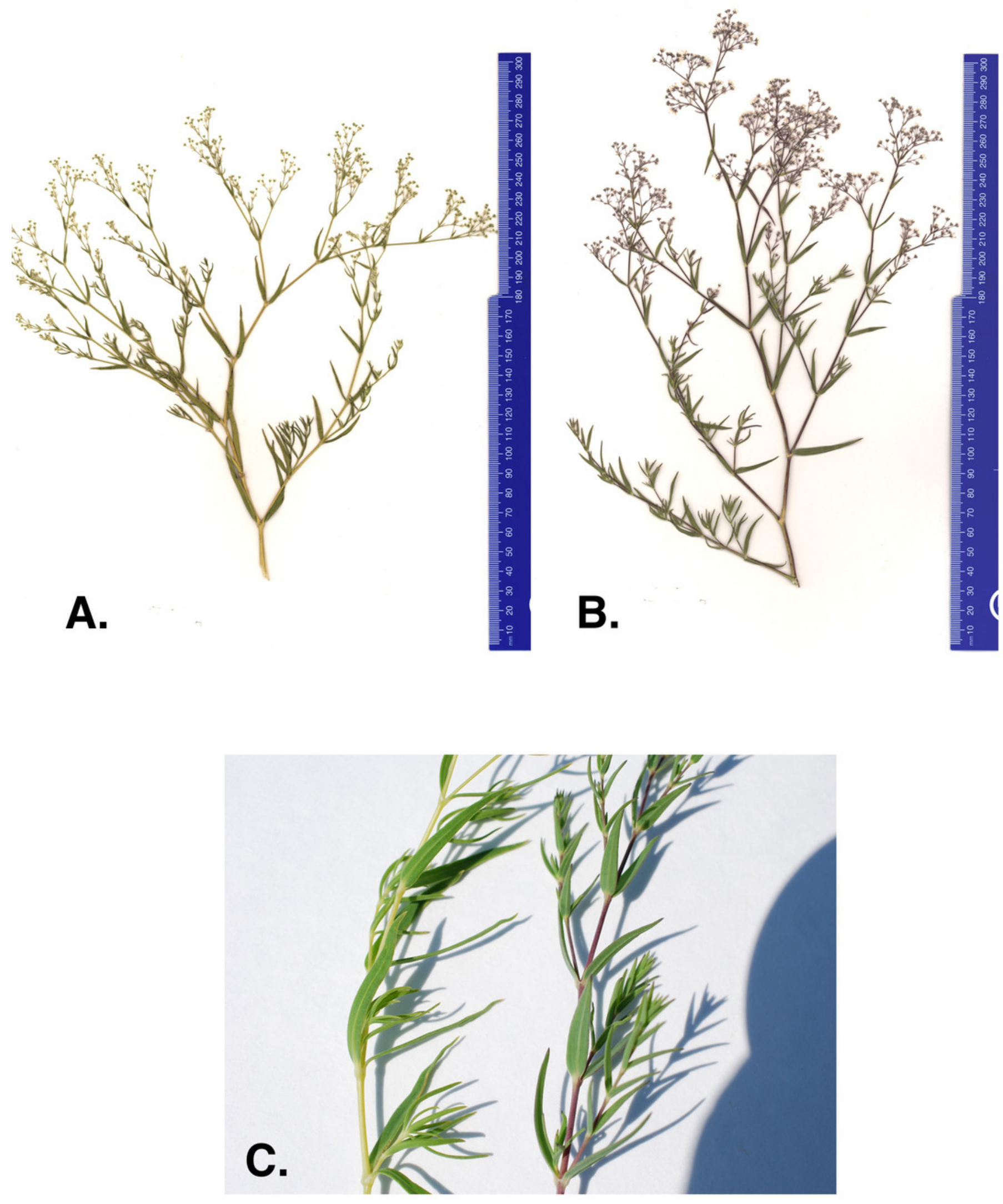
Figure 2

Phylogenetic analysis of the purple and green-yellow baby's breath color morphs in relationship to other Gypsophila species based on the rbcL region.

The evolutionary history was inferred using maximum likelihood methods. For this analysis, we used a Jukes Cantor (JC) model of molecular evolution (Jukes and Cantor, 1969). 


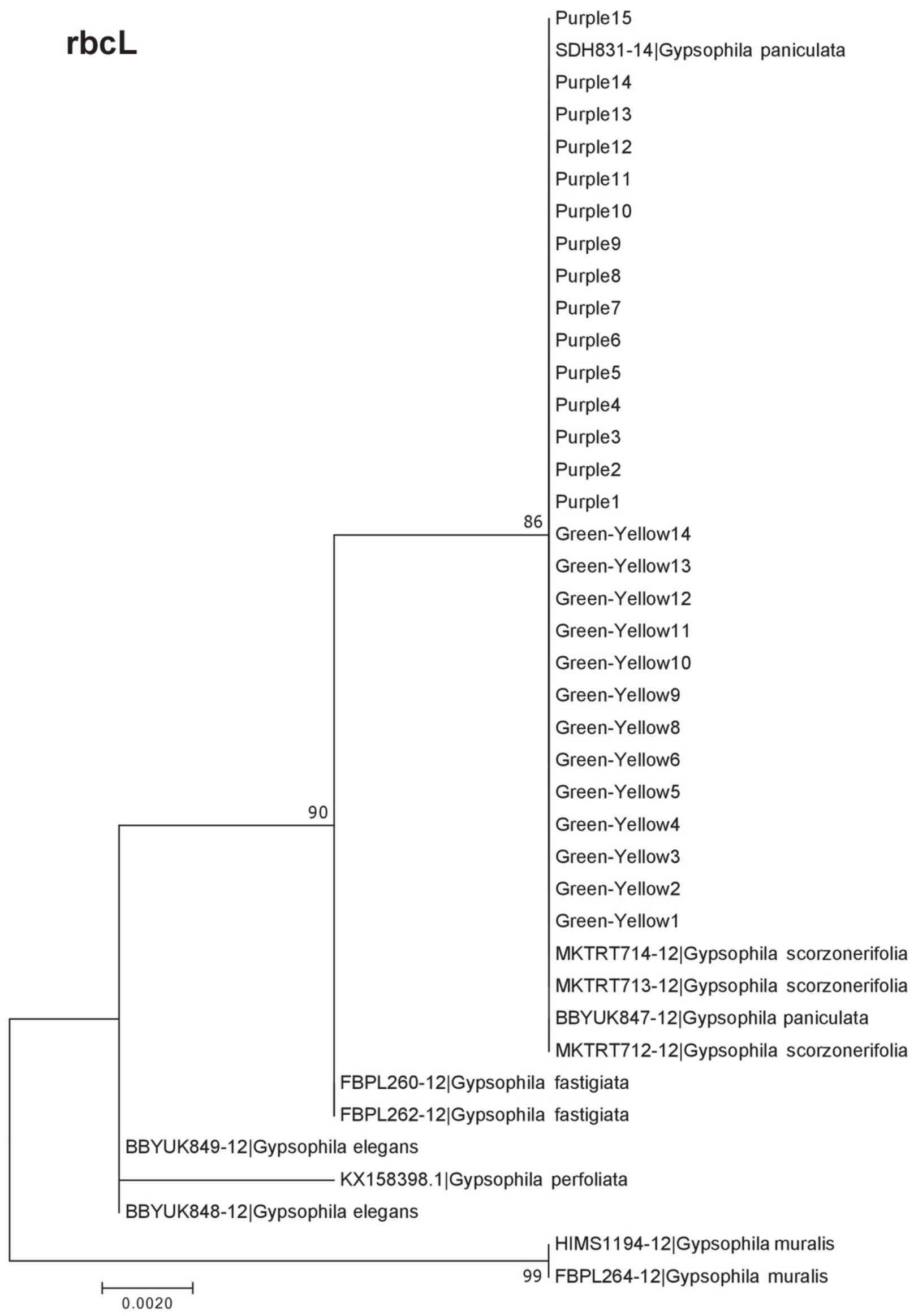


Figure 3

Phylogenetic analysis of the purple and green-yellow baby's breath color morphs in relationship to other Gypsophila species based on the matK region.

The evolutionary history was inferred using maximum likelihood methods. For the analysis, we used a Tamura 3-parameter (T92) model of molecular evolution with uniform distribution (Tamura 1992). 


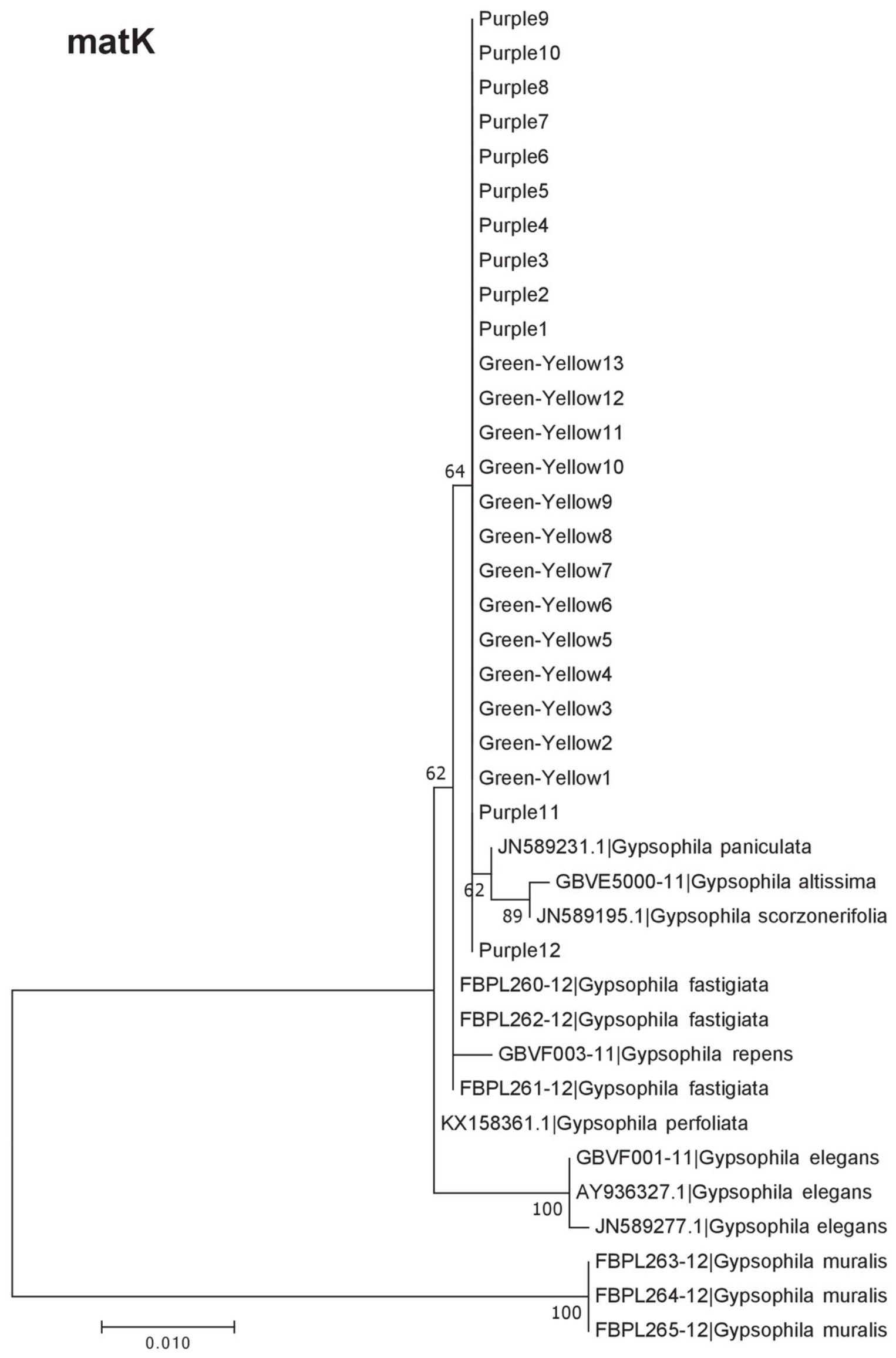


Figure 4

Phylogenetic analysis of the purple and green-yellow baby's breath color morphs in relationship to other Gypsophila species based on the ITS2 region.

The evolutionary history was inferred using maximum likelihood methods. For the analysis we used a Jukes Cantor (JC) model of molecular evolution (Jukes and Cantor, 1969). 


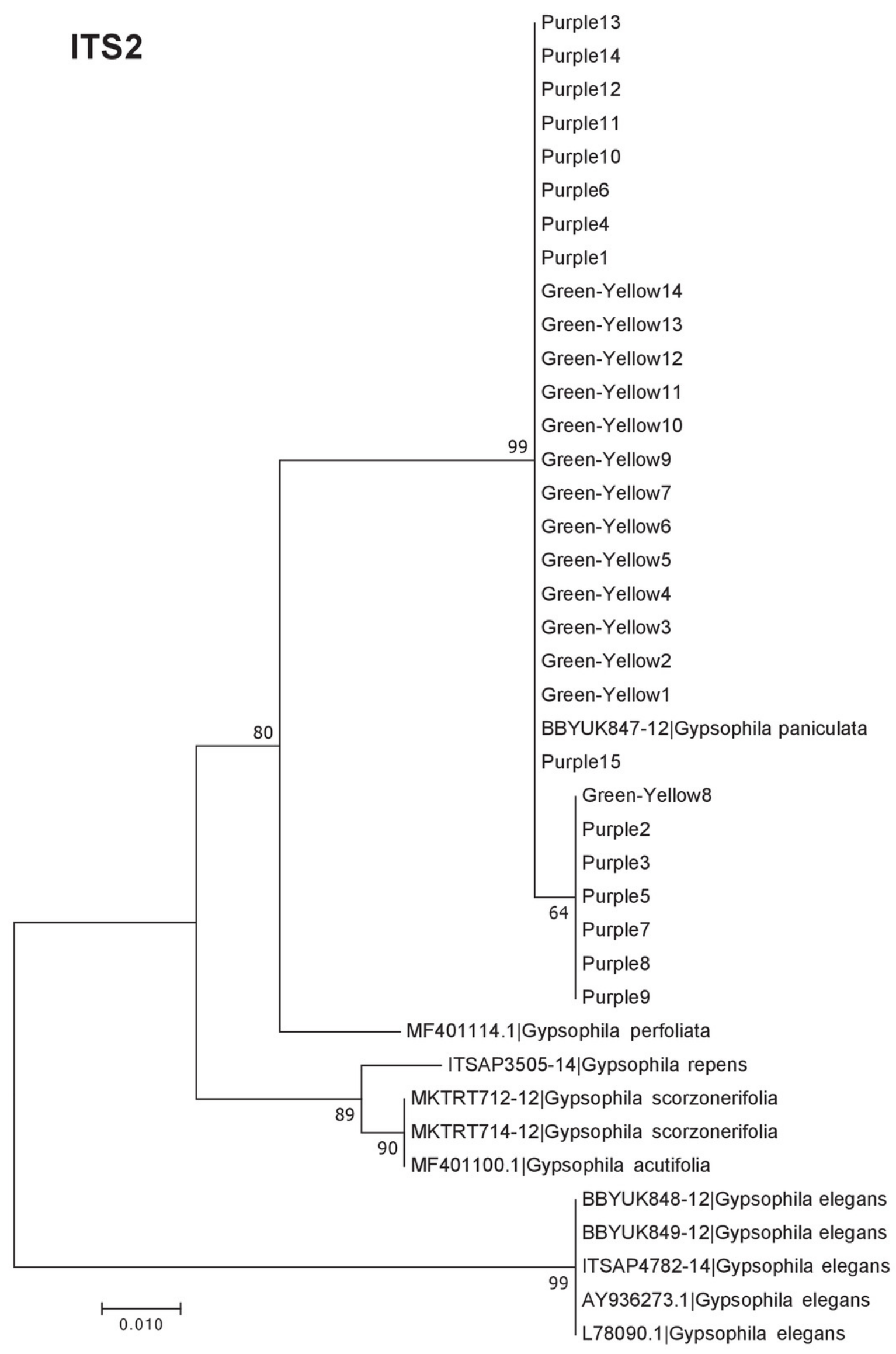




\section{Figure 5}

Phylogenetic analysis of the purple and green-yellow baby's breath color morphs in relationship to other Gypsophila species based on the rbcL, matK, and ITS2 regions combined.

The evolutionary history was inferred using maximum likelihood methods. For the analysis, we used a Tamura 3-parameter (T92) model of molecular evolution with gamma distribution (Tamura 1992). 


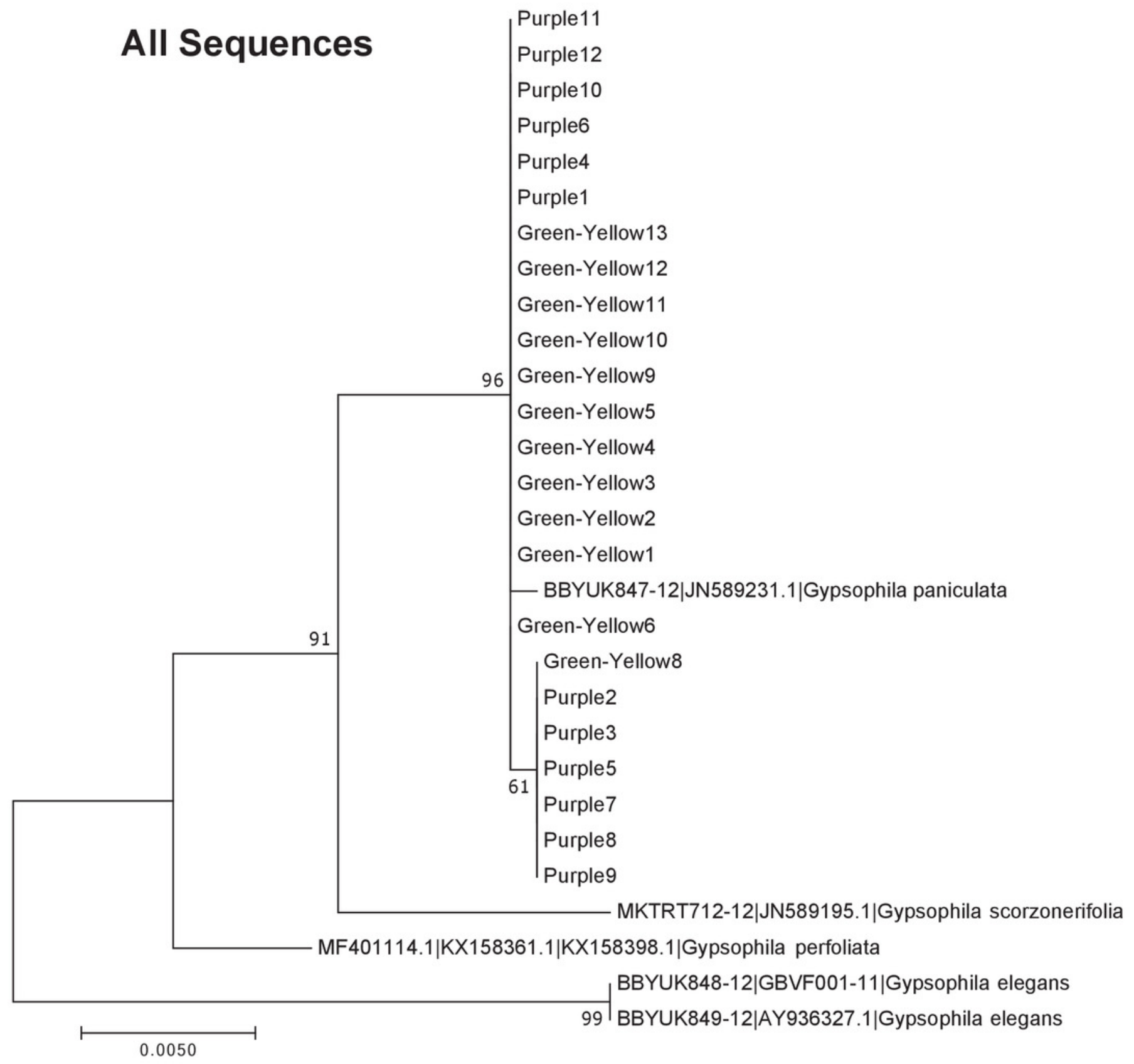


Figure 6

A TCS haplotype network based on rbcL, matK and ITS2 combined for the purple and green-yellow baby's breath color morphs and the $G$. paniculata, $G$. elegans, $G$. perfoliata, and $G$. scorzonerifolia reference sequences.

The size of the ovals correspond to the haplotype frequency. The hash marks represent the number of mutations between each haplotype. 


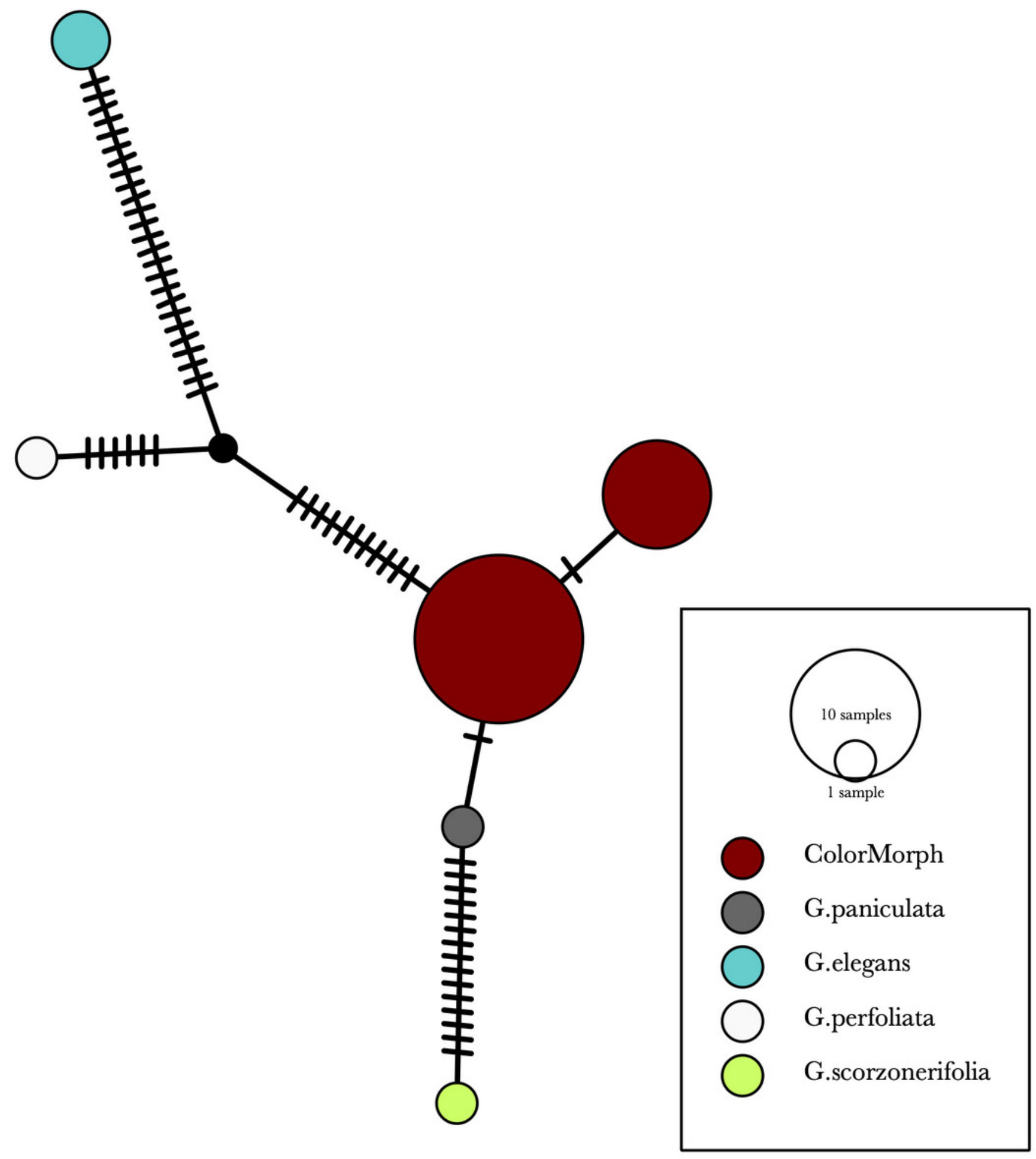




\section{Figure 7}

(A) Green-yellow morph, and (B) common purple morph after 5-months in the GVSU greenhouse.

Note the similarity in taproot structure between the two plants.

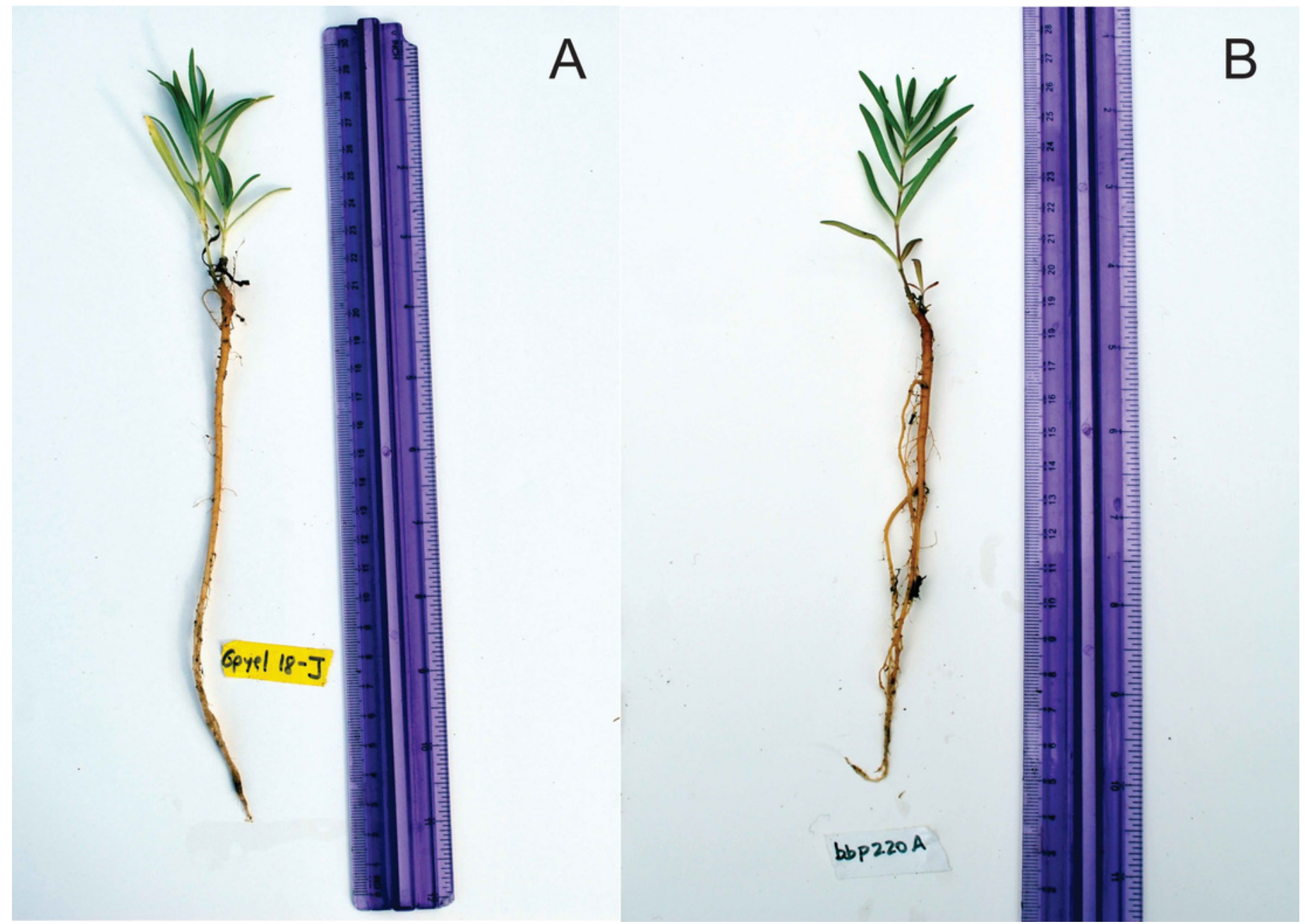

